\title{
Electrical Properties of Carbon Black-Polyethylene Composites
}

\author{
Yoshiaki FujIKUra, Mitsugu KaWAraI, ${ }^{*}$ and Fumihiko OZAKI \\ Department of Polymer Material Engineering, Faculty of Engineering, \\ Yamagata University, 4-3-16 Jonan, Yonezawa, \\ Yamagata 992, Japan
}

(Received January 5, 1989)

\begin{abstract}
The relationship between current density and applied DC electric field was measured for carbon black-polyethylene composite films which contain large amounts of carbon black by volume fraction $\left(v_{\mathrm{f}} \fallingdotseq 0.16-0.28\right)$. The curves relating the current density to the applied DC electric field for the samples show clearly that, at first, the current densities increase in proportion to the applied DC electric fields and then, after the greatest values, they decrease and show negative resistance at high applied DC electric fields. The shapes of the curves are very sensitive to the volume fraction of the carbon black, to a previously applied DC voltage during the sample film molding, and to the density of the matrix polyethylene; yet there is almost no influence due to the particle size of the carbon black or to the melt index of the matrix polyethylene. This finding can be explained well by the tunneling of carriers across the barriers and the generation of Coulomb forces among the carbon black particles.

KEY WORDS Carbon Black-Polyethylene Composite / Current DensityDC Electric Field / High Loading Carbon Black / Negative Resistance / Switching Effect / Shield Material /
\end{abstract}

The problems of the electrical properties of conductive particle-polymer composites have been studied by a number of researchers. Most researchers are chiefly interested in the kind of conductive particle, ${ }^{1-3}$ its volume fraction $\left(v_{\mathrm{f}}\right)$ in a polymer, ${ }^{4-7}$ and the change in resistivity $(R)$ accompanied with temperature raising in a sample, ${ }^{8-11}$ and alternating frequency. ${ }^{12.13}$ The experimental results reported in previous papers were explained well by the thermal expansion of the matrix polymers and the tunneling conduction among the very close conductive particles.

In this paper, we deal with composites of a carbon black (CB) as a conductive component and a polyethylene (PE) as a matrix polymer. The subject of this investigation is the relationship between current density $(J)$ and applied DC electric field $(E)$ for com- posites $^{14-16}$ which contain large amounts of CB, from 0.16 to 0.28 by $v_{\mathrm{f}}$, and so may be regarded as conductors.

The study on the electrical properties of the samples makes it clear that $J$ as a function of $E$ makes a convex curve, that is, there appears a negative resistance after a peak. Experimental results with such noticeable behavior could not be found in previous papers. This electrical phenomenon can be understood in terms of such factors as Coulomb forces being generated among the carbon black particles under the applied electric field. The $J-E$ properties for these composites show a certain kind of switching effect, and so, perhaps they will be useful as a shield material for electromagnetic waves.

\footnotetext{
* Present address: CHINO Co., Ltd., 26-2 Nishishinjuku 1-chome, Shinjuku-ku, Tokyo 163, Japan.
} 


\section{EXPERIMENTAL}

\section{Materials}

A low density polyethylene (LDPE) Sholex M221 supplied by Showa Denko Co., Ltd. with density of $0.915 \mathrm{~g} \mathrm{~cm}^{-3}$ and melt index (MI) of $20 \mathrm{~g} / 10 \mathrm{~min}$ at $190^{\circ} \mathrm{C}$ and a carbon black (CB) Seast V supplied by Tokai Carbon Co., Ltd. with the particle diameter of $62 \mathrm{~nm}$ and a pore volume of $87 \mathrm{ml} / 100 \mathrm{~g}$ dibutyl phthalate (DBP) were used for a matrix polymer and conductive particles.

For comparison, another composite was prepared using a high density polyethylene (HDPE) Sholex 5080, and Seast V CB was used to check the effect of matrix density on $J_{-}$ $E$ relations. The properties of the components are given in Tables I and II.

\section{Sample Preparation}

The $\mathrm{CB}$, powdered and shifted through $\# 120$-mesh screen, was dried at $100^{\circ} \mathrm{C}$ for 50 minutes before blending with $\mathrm{CB}$ in a two roll mill at $140^{\circ} \mathrm{C}$ for ten minutes. The volume fraction of $\mathrm{CB}$ varied from 0.16 to 0.28 . A $0.6 \mathrm{~mm}$ thick film was molded from each composite by a hot-press at $140^{\circ} \mathrm{C}$ and at $150^{\circ} \mathrm{C}$ for ten minutes for LDPE and HDPE respectively, and then they were quenched in ice water.

\section{RESULTS AND DISCUSSION}

\section{Relationship between Resistivity and CB Content}

Diagrams of the experimental apparatus used to measure the $R$ between the opposing faces of the composite films are shown in Figure 1. In these diagrams, 'Sample' refers to the composite films blended with a $\mathrm{PE}$ and a $\mathrm{CB}$, and 'Electrode' refers to a copper plate. The dimensions of each test piece in the $R$ measurement is $0.6 \mathrm{~mm}$ in thickness and $30.0 \mathrm{~mm}$ in both width and length. Both sides of the films are coated with silver paste so that contact is just made between each electrode and the composite film. The relationship between $R$ and $\mathrm{CB}$ volume fraction for the test
Table I. Properties of the polyethylene used in this experiment

\begin{tabular}{lccc}
\hline & \multicolumn{1}{c}{ MI at $190^{\circ} \mathrm{C}$} & Density \\
\cline { 2 - 3 } & $\mathrm{g} / 10 \mathrm{~min}$ & & $\mathrm{~g} \mathrm{~cm}^{-3}$ \\
\hline LDPE (Sholex M221) & 20 & 0.915 \\
HDPE (Sholex 5080) & 7.8 & 0.949 \\
\hline
\end{tabular}

Table II. Properties of the carbon black used in this experiment

\begin{tabular}{lcc}
\hline \multirow{2}{*}{ Filler } & Particle diameter & Absorption of DBP $^{\mathrm{a}}$ \\
\cline { 2 - 3 } & $\mathrm{nm}$ & $\mathrm{ml} / 100 \mathrm{~g}$ \\
\hline CB (Seast V) & 62 & 87 \\
\hline
\end{tabular}

a Dibutyl phthalate.

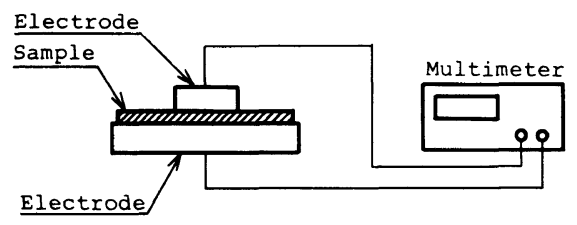

(a)

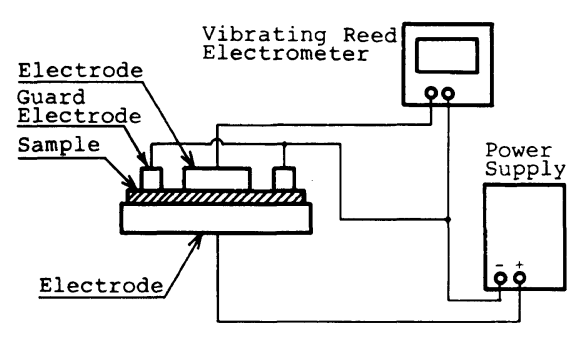

(b)

Figure 1. Diagrams of the apparatus used to measure the resistivity of the composite samples: (a) for low resistance measurements; (b) for high resistance measurements. Two copper electrodes are used, giving a large heat capacity.

samples is shown in Figure 2. In the range of the $\mathrm{CB}$ volume fraction up to a critical volume fraction $\left(v_{\mathrm{fc}}\right)$, the $R$ is nearly constant, being that of the PE itself used in the matrix. The extreme drop in $R$ at a certain $v_{\mathrm{f}}$ is believed to be due to network formations among the $\mathrm{CB}$ 


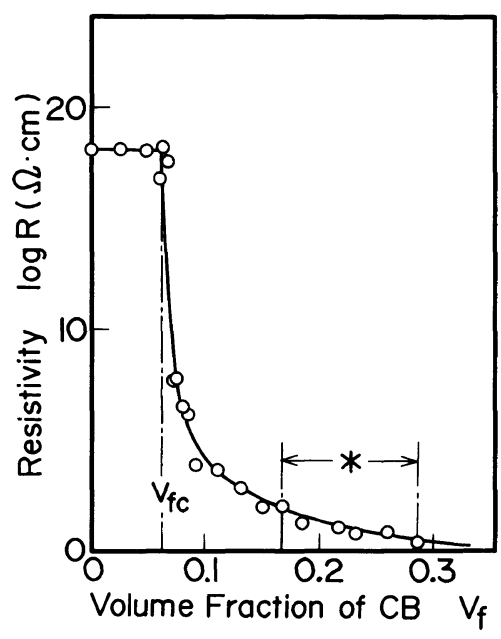

Figure 2. Relationship between resistivity and volume fraction for a LDPE-CB composite, at $20^{\circ} \mathrm{C}$. LDPE, Sholex M221; CB, Seast V, $v_{\mathrm{fc}}=$ critical volume fraction. ' $*$ ' indicates the range of the $J-E$ measurement.

particles. The $v_{\mathrm{f}}$ of the $\mathrm{CB}$ is determined as follows:

$$
v_{\mathrm{f}}=\frac{v_{\mathrm{CB}}}{v_{\mathrm{PE}}+v_{\mathrm{CB}}}
$$

where $v_{\mathrm{PE}}$, volume of the matrix $\mathrm{PE}$ $v_{\mathrm{CB}}$, volume of the $\mathrm{CB}$

\section{Current-Voltage Property}

This investigation was interested in the composite as a conductor with the $v_{\mathrm{f}}$ of the $\mathrm{CB}$ in the range of 0.16 to 0.28 . The range of measurements is indicated by the ' $*$ ' in Figure 2. According to convenience of the electric power source, the dimensions of each test sample in the $J-E$ measurement were $0.6 \mathrm{~mm}$ in thickness and $3.0 \mathrm{~mm}$ in both width and length.

A diagram of the experimental apparatus used to measure the $J-E$ property is shown in Figure 3. In previous experiments with this problem, researchers generally applied a pulsed electric field so as to avoid the generation of heat in the test sample. However, we decided to use a DC electric field in this study. If a pulsed electric field is applied to a composite,

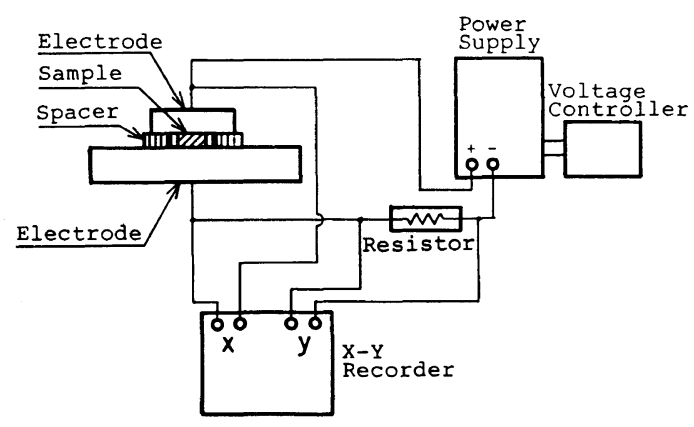

Figure 3. Diagram of the apparatus used to measure the $J-E$ property. The two copper electrodes have a large heat capacity.

the $J-E$ property may show a transient phenomenon in the $\mathrm{R}-\mathrm{C}$ circuit formed among the gaps of $\mathrm{CB}$ particles with electrostatic capacity. It was for this reason that a DC electric field was used in this experiment. The problems of heat generation were solved through the use of large-sized copper electrodes with high heat capacity as opposed to small-sized test piece, and through the constant checking of sample temperatures by the thermocouples. Such checking made it clear that the sample temperature rose only slightly during the experiment.

The $J-E$ experimental results for the test samples are plotted in Figure 4. According to the results, the $J$ increases in proportion to the $E$ in the low $E$ region, and then, after the $J$ peaks at a certain applied $E$, it gradually decreases, that is, shows negative resistance $(\mathrm{d} J / \mathrm{d} E<0)$. The results of the experiment are as follows:

i) each one of the $J-E$ curves reaches a peak at a certain $E$, followed by negative resistance $(\mathrm{d} J / \mathrm{d} E<0)$.

ii) as the $v_{\mathrm{f}}$ increases, the $J$ increases, and the $J-E$ curve becomes sharper.

iii) the $J-E$ property is a reproducible and reversible phenomenon within the breakdown E.

Since no theoretical study has been conducted on large amounts of CB distribution in a polymer matrix, we present the following 


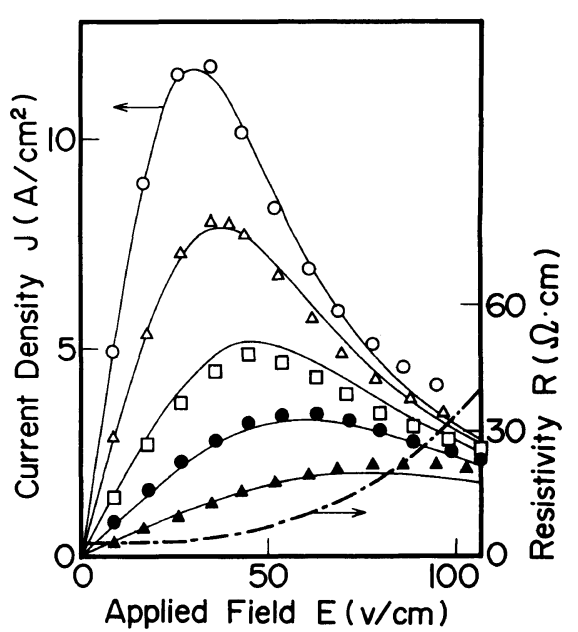

Figure 4. $J-E$ properties are plotted based on experimental results, using LDPE (Sholex M221), at $20^{\circ} \mathrm{C}$, with the following CB (Seast V) volume fractions: $v_{\mathrm{f}}$, 0.165 ( $\Delta$ ), 0.198 (○), $0.229(\square), 0.257(\triangle), 0.284(\bigcirc)$, and drawn based on experimental equation (2) by fine lines. The broken line represents an $R-E$ curve $\left(v_{\mathrm{f}}=\right.$ 0.257 ) converted from the $J-E$ curve.

experimental equation which express the experimental results of the relationship of the $v_{\mathrm{f}}$ to the $J$ and $E$ :

$$
J=\frac{E}{a \times E^{3}+b \times c^{v_{\mathrm{f}}}}
$$

where

$$
\begin{array}{ll}
J, \text { current density } & a=3.03 \times 10^{-5} \\
E, \text { applied field } & b=1053 \\
v_{\mathrm{f}}, \text { volume fraction of CB } c=1.59 \times 10^{-10}
\end{array}
$$

The terms $a \times E^{3}$ and $b \times c^{v_{\mathrm{f}}}$ are resistances which depend on the applied DC electric field and the dispersion of $\mathrm{CB}$ in the composite, respectively. Since this experimental equation fairly well fits with the experimental results shown by the fine line in Figure 4 , it will be practical and useful for the members of a planning group.

The broken line in Figure 4 represents an $R-$ $E$ curve $^{17}\left(v_{\mathrm{f}}=0.257\right)$ converted from the $J-E$ curve.

In this curve, the $R$ is nearly constant up to the peak of the $J-E$ curve, after which it

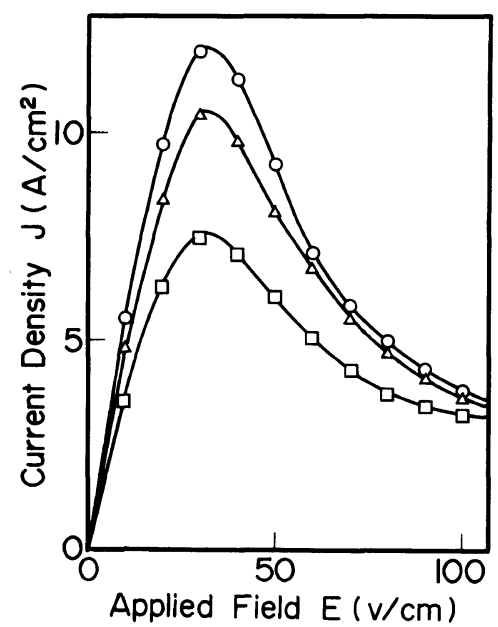

Figure 5. $J-E$ properties of the composite with previously applied $\mathrm{DC}$ electric field, at $20^{\circ} \mathrm{C}$. Components: LDPE, Sholex M221; CB, Seast V. $v_{\mathrm{f}}, 0.284$. Voltage: $\bigcirc$, $0 ; \triangle, 5 ; \square, 7$.

increases gradually till the breakdown $E$.

From our other experiment, it was also recognized that the $J$ maintained a nearly constant value under a constantly applied $E$, and the sample temperature barely changed during the measurement.

\section{Effects of a Previously Applied Electric Field}

A DC electric field was applied to the composite films for one minute when the films were being molded, after which the films were quenched in ice water. The DC electric fields were applied by $0,5,7$ volts to the different composite films. The $J-E$ properties of the samples are shown in Figure 5. It was recognized from the results that as the applied voltage at molding time increased, the peak of the $J-E$ curve decreased. It is certain that the $R$ of the composite film increased with applied DC electric field when it was being molded and quenched.

The dispersing CB particles in a matrix PE can be supposed to be in one of two states. In one, contact among the CB particles conducts an electric current by a conduction electron. In the other, infinitesimal gaps among the sepa- 


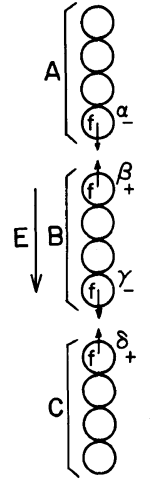

(a)

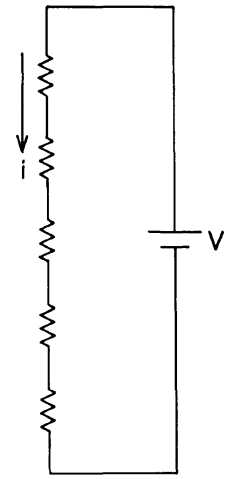

(b)
Figure 6. Electric equivalent circuit of dispersed CB particles in a composite under a DC electric field. A-C, CB particles.

rated $\mathrm{CB}$ particles may conduct an electric current by a tunnel effect. The electrical operation of the CB particles in a composite is shown by the electric equivalent circuit in Figure 6. Separated CB particles, such as $\alpha \beta$ and $\gamma \delta$, may have electrostatic capacity; therefore the $\mathrm{CB}$ particles may charge as in the circuit in the figure. The charged $\mathrm{CB}$ particles in molten matrix PE may generate Coulomb attractive forces among the separated particles $(\alpha-\beta$ and $\gamma-\delta)$ and repulsive forces among the contacting particles $(\beta-\gamma)$ and thus the $R$ may decrease according to those conditions, but these conditions may increase in the $R$. Total $R$ under the conditions as described above may increase mainly due to a relaxation in pressure at contacting surface of the $\mathrm{CB}$ particles and a break in filaments interconnected by CB particles.

These effects may appear markedly in molten composites than in solid ones, and also in proportion to the applied voltage in each state. From these reasons, negative resistance is clearly generated by Coulomb attractive force at high applied voltages in solid samples.

\section{Effects of the Density of the Matrix PE}

The influence of the density of the matrix PE on the $J-E$ property of the composites was

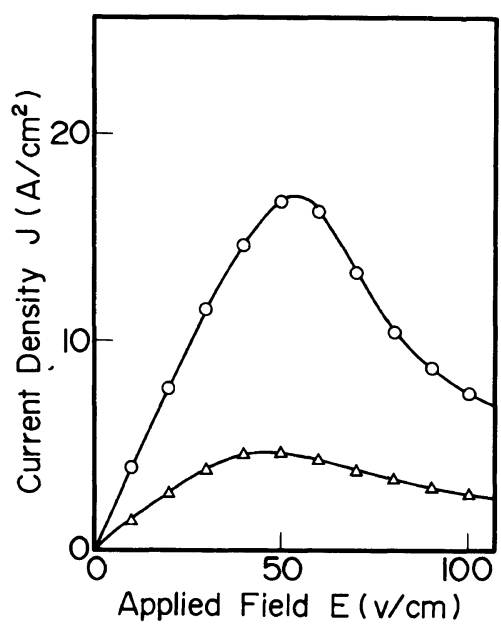

Figure 7. $J-E$ properties for different densities of the two matrices in composite, at $20^{\circ} \mathrm{C}$. Matrix: $\mathrm{O}, \mathrm{HDPE}$ (Sholex 5080); $\triangle$, LDPE (Sholex M221); CB, Seast V; $v_{\mathrm{f}}$, 0.229 .

investigated. The LDPE with $0.915 \mathrm{~g} \mathrm{~cm}^{-3}$ and the HDPE with $0.949 \mathrm{~g} \mathrm{~cm}^{-3}$ were selected as the densities of different matrices. Based on the experimental results, the $J$ increases as the density of the matrix PE becomes higher. The results are shown in Figure 7. What is obvious on comparing the two curves is that when HDPE is selected as the matrix, the $J$ increases by about three times that when LDPE is selected. ${ }^{18}$ It is very difficult for CB particles to enter the crystals. Therefore, as the CB particles gather in a noncrystalline region, the real $v_{\mathrm{f}}$ of the $\mathrm{CB}$ in the amorphous parts of the HDPE may become larger than that of the LDPE. The result, as described above, is that the $J-E$ properties of the HDPE matrix behave as a large $v_{\mathrm{f}}$ value in LDPE matrix.

The maximum current density $\left(J_{\max }\right)$ with the calculated volume fraction $\left(v_{\mathrm{f}}{ }^{\prime}\right)$ based on the assumption that the CB particles in LDPE (Figure 4) and HDPE (Figure 7) gathered only noncrystalline region is shown in Figure 8. From the figure, it is recognized that each $J_{\max }$ is well related to the increase in $v_{\mathrm{f}}{ }^{\prime}$.

The effects of the MI of the PE and the particle size of the $\mathrm{CB}$ on the $J-E$ properties of 


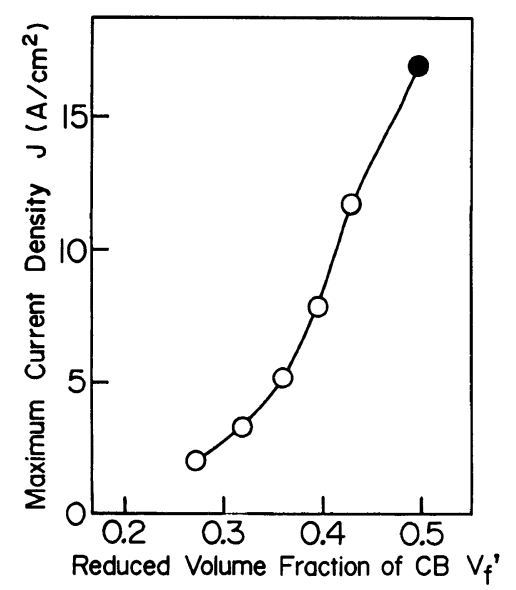

Figure 8. Maximum current density with calculated volume fraction regarded as the $\mathrm{CB}$ particles are dispersed only noncrystalline region. $\bigcirc$, for LDPE in Figure 4; , for HDPE in Figure 7.

the composites are basically none; that is, differences in the MI and the particle size have no effect on the $J-E$ properties. Therefore, the results for these situations are omitted in this paper.

\section{CONCLUSION}

Composites of PE and large amounts of $\mathrm{CB}$ increase in $J$ according to Ohm's law, with an low applied DC electric field, and then $J$ decreases after a peak. The decrease in $J$ at higher applied fields may be caused by contacting pressure relaxation and break in CB chains induced by Coulomb attractive forces among the separated CB prticles. The $J-E$ property in this investigation shows a switching effect. The simple experimental equation provided by us can be applied to this sort of composite planning. The $J-E$ properties are strongly influ- enced by the $v_{\mathrm{f}}$ of the $\mathrm{CB}$, a previously applied DC electric field during the sample molding, and the density of the matrix PE. The differences in size of the CB particles and in MI of the matrix PE have little influence on the $J-E$ property. The influence of matrix polymer species, the degree of crystallinity in detail, and the like, on the $J-E$ property should be investigated in future.

\section{REFERENCES}

1. A. Malliaris and D. T. Turner, J. Appl. Phys., 42, 614 (1971).

2. J. B. Donnet and A. Voet, "Carbon Black," Marcel Dekker, New York, N.Y., 1976, Chapter 3.

3. M. Sumita, E. Jojima, H. Aida, K. Miyasaka, and K. Ishikawa, Kobunshi Ronbunshu, 40, 203 (1983).

4. S. M. Aharomi, J. Appl. Phys., 43, 2463 (1972).

5. C. Rajagopal and M. Satyam, J. Appl. Phys., 49, 5536 (1978).

6. D. M. Bigg, Polym. Eng. Sci., 19, 1188 (1979).

7. K. Miyasaka, K. Watanabe, E. Jojima, H. Aida, M. Sumita, and K. Ishikawa, J. Mater. 'Sci., 17, 1610 (1982).

8. S. Saito, H. Sasabe, T. Nakajima, and K. Yada, $J$. Polym. Sci., A-2, 6, 1297 (1968).

9. K. Ohe and Y. Naito, Jpn. J. Appl. Phys., 10, 99 (1971).

10. F. Bueche, J. Appl. Phys., 44, 532 (1973).

11. R. D. Sherman, L. M. Middleman, and S. M. Jacobs, Polym. Eng. Sci., 23, 36 (1983).

12. F. Bueche, J. Polym. Sci., Polym. Phys. Ed., 11, 1319 (1973).

13. K. T. Chung, A. Sabo, and A. P. Pica, J. Appl. Phys., 53, 6867 (1982).

14. E. K. Sichel, J. I. Gittleman, and P. Sheng, Phys. Rev. B, 18, 5712 (1978).

15. S. H. Kwan, F. G. Shin, and W. L. Tsui, J. Mater. Sci., 15, 2978 (1980).

16. F. J. Baltacalleja, T. A. Ezquerra, D. R. Rueda, and J. A. Lopez, J. Mater. Sci. Lett., 3, 169 (1984).

17. J. G. Simmons, J. Appl. Phys., 34, 1793 (1963).

18. J. Mayer, Polym. Eng. Sci., 13, 462 (1973). 